\title{
O nascimento do saber infodemiológico: A ciência da gestão de infodemias
}

\author{
The birth of infodemiology: the science of managing \\ infodemics
}

\begin{abstract}
André Arias a,*
RESUMO: Este artigo examina os estágios iniciais da instauração de um novo campo do saber denominado de infodemiologia. Em particular, procura traçar o percurso do engendramento da infodemiologia enquanto o discurso científico de gestão da infodemia que acompanha a pandemia do novo coronavírus. Esse novo campo do saber está sendo desenvolvido, estruturado e publicizado pela Information Network for Epidemics vinculada à OMS, ONU e UNESCO. Com o surto informacional que acompanha a condição pandêmica vigente, o discurso infodemiológico, de domínio periférico da epidemiologia, parece se inscrever aos poucos na malha dos saberes científicos, dos protocolos de governança e do exercício de poder. A proposta é fazer a análise do discurso infodemiológico em sua articulação com as forças políticas da economia digital e do capitalismo de plataforma vigente.
\end{abstract}

Palavras-chave: Infodemiologia; Infodemia; Pandemia; Capitalismo de Plataforma.

ABSTRACT: This article investigates the initial stages of a new field of knowledge called infodemiology. Particularly, it seeks to trace the development of infodemiology as the scientific discourse of managing infodemic. This new field of knowledge is being developed, structured, and publicized by the Information Network for Epidemics, a department of WHO, UN, and UNESCO. As the informational outbreak surges, the infodemiological discourse slowly enters the fabric of scientific knowledge, governance protocols, and power institutions. The proposal here is to analyze the infodemiological discourse in its articulation with the political forces of the digital economy and the current platform capitalism.

Keywords: Infodemiology; Infodemics; Pandemics; Platform Capitalism.

a Centre for Interdisciplinary Studies in Society and Culture, Concordia University, Montreal, Canadá.

*Correspondência para/Correspondence to: André Arias. E-mail: andre.fogli@gmail.com.

Recebido em/Received: 23/04/2021; Aprovado em/Approved: 16/06/2021.

Artigo publicado em acesso aberto sob licença CC BY 4.0 Internacional () (i) 


\section{INTRODUÇÃO}

Neste artigo, examino os estágios iniciais da instauração de um novo campo do saber denominado de infodemiologia. Particularmente, procuro explorar o percurso que fez com que a infodemiologia emergisse como o discurso científico mais adequado para gerir a infodemia que acompanha a pandemia do novo coronavírus. Esse novo campo do saber está sendo desenvolvido pela Information Network for Epidemics (EPI-WIN) da Organização Mundial de Saúde (OMS), com a cooperação da Organização das Nações Unidas (ONU) e da Organização das Nações Unidas para a Educação, a Ciência e a Cultura (UNESCO), além de instituições e setores parceiros a nível local, regional ou global, ligados a governos e Estados ou não. Com a condição infodêmica vigente, o discurso infodemiológico, de domínio periférico da epidemiologia, parece se inscrever aos poucos na malha dos saberes científicos, dos protocolos de governança e do exercício de poder.

Examino esse material por meio de um crivo muito específico. Qual seja: detectar os pontos de articulação entre saber e poder. Como explicitado por Roberto Machado (1978, p. 11), saber e poder se articulam "não em termos de exterioridade ou de justaposição, em que o poder se apropriaria de uma neutralidade científica e a utilizaria segundo objetivos que lhe são extrínsecos, mas de imanência: a dimensão política é constitutiva da existência dos discursos". Isso significa que o enunciado científico está, desde sua gênese, entrelaçado com as infraestruturas políticas de sua atualidade geohistórica. Esses discursos procuram extrair um rendimento específico dos indivíduos e das populações. Em acontecimentos históricos de magnitude similar ao da pandemia da Covid-19, a ciência médica cumpriu essa função de municiar os poderes com as ferramentas e procedimentos mais eficientes para intervir na dinâmica social, sem contar os eventos em que ela própria representou e exerceu o poder. ${ }^{1}$

\footnotetext{
${ }^{1}$ Cito três títulos que abordam essa relação de imanência entre saber e poder, em especial o saber médico e o desenvolvimento de técnicas de intervenção política no corpo e na conduta dos governados e na distribuição espaço-temporal dos corpos nas instituições e na cidade. Um exemplo clássico é aquele da peste abordado por Michel Foucault em Vigiar e Punir (1999), no início do capítulo em que ele introduz o conceito e caracteriza o dispositivo do panóptico. Neste caso, Foucault descreve em minucias como o saber médico forneceu e implementou todo um protocolo extremamente criterioso de práticas necessárias para o controle dos corpos durante a peste para assegurar a saúde da população da cidade. Em nome da vida, sistematiza-se um dispositivo de poder fundamentado na legitimidade e na autoridade científica do saber médico. Um outro acontecimento paradigmático é descrito por Sidney Chalhoub em $\mathrm{A}$ cidade febril (1996). Chalhoub narra como o saber médico pensou as epidemias de febre amarela que grassaram nos principais centros da economia colonial-escravista do continente americano (Cuba, São Domingos/Haiti, sul dos Estados Unidos e Brasil). Chalhoub quer demonstrar como o discurso científico era indissociável da política, no sentido de que a ciência sanitarista era (é) um instrumento político que deve ser analisado não só por sua coerência interna, mas por suas consequências e efeitos políticos. Como Chalhoub esclarece, o saber médico tinha a finalidade, verbalmente enunciada, por exemplo, nos plenários da Intendência do Rio de Janeiro, de diretamente intervir na lógica de urbanização da capital do Império. O livro esmiúça o desenvolvimento histórico deste discurso ao longo de todo o século XIX e início do XX, e suas implicações e aplicações políticas na época e sua reverberação nas políticas do presente. Chalhoub percorre no livro a disputa aberta entre os diferentes discursos médicos (higienistas $e$ sanitaristas, sobretudo, mas também holopatas, homeopatas etc.) que discorriam particularmente em dois espaços, nas tribunas dos principais jornais da época e nas instâncias de decisão política, onde muitos de seus integrantes eram, claro, médicos. O discurso dos médicos higienistas sairia vitorioso, ao final, por exemplo, na indicação de um médico higienista para o cargo de prefeito do Distrito Federal, Barata Ribeiro (nome de uma das maiores e mais importantes ruas da zona rica da cidade), que projetou a reurbanização
} 
É justamente a maneira como essa intervenção é pensada e exercida que me interessa apreender da literatura de referência sobre a infodemiologia. Em específico, interessame descrever como essa vontade de intervir é construída entretecendo saberes, a princípio, pouco próximos, quais sejam, o saber médico, epidemiológico, a teoria da comunicação e da informação, a análise de comportamento, behaviorismo, bem como a ciência da computação e de dados. Essa articulação está presente tanto nos documentos publicados pela EPI-WIN quanto no discurso infodemiológico que a precede - o texto inaugural da infodemiologia, que abordo adiante, é de 2002.

A questão da infodemiologia é saber como influenciar o comportamento das populações e dos indivíduos, tendo em vista um rendimento imediato, no caso o comportamento mais adequado para o controle da pandemia e da infodemia da Covid19 (fica em casa, use máscara), e, como a própria EPI-WIN deixa claro, das pandemias e infodemias que virão. Os procedimentos instaurados pela infodemiologia são mobilizados para que se coloque em marcha uma mudança comportamental necessária para refrear a curva do surto informacional, da mesma maneira que a epidemiologia oferece as técnicas e as estratégias mais eficazes para que a curva de transmissão do vírus seja refreada. Portanto, saber e poder: esta infra-relação compõe o crivo pelo qual procuro examinar o nascimento do saber infodemiológico e fazer a análise do seu discurso.

\section{A INFODEMIA}

Como disse na introdução, a Information Network for Epidemics (EPI-WIN) é a rede responsável, dentro da OMS, para estruturar, estabelecer e implementar o saber infodemiológico globalmente. A EPI-WIN é uma iniciativa da Organização Mundial de Saúde (OMS), com a cooperação da Organização das Nações Unidas (ONU) e da Organização das Nações Unidas para a Educação, a Ciência e a Cultura (UNESCO), além de instituições e setores parceiros a nível local, regional ou global, ligados a governos e Estados ou não. Além da missão de consolidar a infodemiologia como a ciência de gerenciamento de infodemias, seja organizando Conferências, cursos de treinamento em infodemiologia, ou publicando documentos sobre a disciplina, a EPI-WIN trabalha também com o intuito de traduzir e publicizar esses conhecimentos para as instituições de governo e da sociedade civil interessadas em ter um parâmetro homogêneo e adaptável para efetivação de políticas públicas.

Para a EPI-WIN, o saber infodemiológico tem um objeto de investigação preciso: a infodemia, a pandemia informacional que se combinou à pandemia do novo coronavírus. A OMS e a ONU reconheceram cedo o risco de letalidade que o fenômeno carrega. Os Secretários-Gerais de ambas as Organizações, Tedros Adhanom Ghebreyesus e António Guterrez, declararam categoricamente sua preocupação com

do centro do Rio de Janeiro que expulso violentamente a classe perigosa e pobre que habitavam os cortiços do centro. 
a proliferação descontrolada de informações. ${ }^{2}$ Em 19 de maio de 2020, a $73^{\circ}$ Assembleia da OMS apresentou uma resolução na qual convocava todos os Estados-membros a se comprometerem com as propostas ali contidas para o devido enfrentamento da pandemia. ${ }^{3}$ Entre os quinze itens dispostos, o item seis aponta, precisamente, para a necessidade de "tomar medidas de combate à desinformação e a informação imprecisa, além de ciberatividades maliciosas". ${ }^{4}$ Seguindo essa diretriz, a EPI-WIN foi instaurada para ser a plataforma de gestão da infodemia, o setor responsável por dirigir e coordenar um programa de prática e de pesquisa com o intuito de sedimentar as bases epistemológicas da nova ciência.

Desde então, a EPI-WIN organiza uma série de encontros online, com especialistas de várias disciplinas e com múltiplos setores da sociedade, para pensar conjuntamente como lidar com o problema. Além disso, a rede vem consolidando e publicando continuamente um rico material para alinhar uma metodologia e uma linguagem comum e replicável. O objetivo é oferecer aos Estados-membros, à sociedade e a demais agentes interessados (plataformas, mídia, mercado, comunidades religiosas, organizações não-governamentais) propostas de protocolos, medidas e políticas públicas a serem efetivadas para que seja possível uma cooperação internacional para a gestão da infodemia.

Pois bem, o que vem a ser a infodemia? Num texto coautorado por um grupo de pesquisadore/as vinculado à EPI-WIN, a infodemia é definida assim:

\footnotetext{
o excesso de informação - algumas precisas e outras não - que ocorre durante uma epidemia. De maneira semelhante a uma epidemia, ela se espalha entre os seres humanos através de sistemas de informação digital e física. Torna difícil para as pessoas encontrar fontes e orientação confiáveis quando precisam" (Tangcharoensathien et al, 2020, p. 2).5
}

Em um dos primeiros documentos oficiais a respeito, veiculado pela Organização PanAmericana da Saúde, encontra-se a seguinte definição:

O que é infodemia? (...) Um excesso de informações, algumas precisas e outras não, que tornam difícil encontrar fontes idôneas e orientações

\footnotetext{
2 Em fevereiro de 2020, antes da OMS declarar o estado de pandemia, Ghebreyesus afirmou: "Não estamos apenas combatendo uma epidemia, estamos combatendo uma infodemia". Acessado em: 30 mai. 2020. Disponível em: https://www.who.int/director-general/speeches/detail/director-general-s-remarks-at-themedia-briefing-on-2019-novel-coronavirus---8-february-2020. Pouco tempo depois, em março, o Secretário-Geral da ONU, no vídeo de lançamento da agência de checagem de fato Verificado, disse: "enquanto a pandemia da Covid 19 se alastra, outro vírus tem infectado nossas vidas: a informação imprecisa (misinformation)". Acessado em: 30 mai. Disponívem em: https://www.un.org/africarenewal/news/coronavirus/covid-19-united-nations-launches-global-initiativecombat-misinformation.

3 A preocupação da ONU com o tema da desinformação e das fake news e o modelo de negócios das plataformas e da economia digitais não são novos. Na ata do encontro do Conselho de Direitos Humanos da Assembleia Geral de 2018, já consta o desejo de regular as plataformas de maneira a coibir determinadas práticas. Ademais, a UNESCO publicou diversos documentos importantes sobre questões relativas à internet, jornalismo e desinformação e temas congêneres.

${ }_{4}^{4}$ Acessado em em 1 de mar. 2020. Disponível: https://apps.who.int/gb/ebwha/pdf_files/WHA73/A73_R1en.pdf

5 Todas as traduções das referências utilizadas ao longo do texto que não encontram versão em português são de minha tradução.
} 
confiáveis quando se precisa. A palavra infodemia se refere a um grande aumento no volume de informações associadas a um assunto específico que podem se multiplicar exponencialmente em pouco tempo devido a um evento determinado, como a pandemia atual. Nessa situação, surgem rumores e desinformação, além da manipulação de informações com intenção duvidosa. Na era da informação, esse fenômeno é amplificado pelas redes sociais e se alastra mais rapidamente, como um vírus (OPAS, 2020, P. 2).

Contudo, o primeiro artigo que a mobiliza o conceito é de 2003, quando a síndrome respiratória aguda grave (SARS) reapareceu como uma ameaça epidêmica. Rothkopf (2003) logo percebeu que a epidemia de SARS era uma dupla epidemia.

\begin{abstract}
A SARS não é a história de uma epidemia, mas duas, e a segunda epidemia, a que escapou em grande parte das manchetes, tem implicações muito maiores do que a própria doença. Isso porque não é a epidemia viral, mas sim uma "epidemia de informação" que transformou a SARS [...] em um desastre econômico e social global. A SARS [...] sofreu um pesado tributo com mais de 7.100 vítimas relatadas em todo o mundo e mais de 500 mortes relatadas até agora. Mas as consequências da "epidemia" relacionada foram mais abrangentes do que a doença subjacente e, muito possivelmente, mais dispendiosas, afetando a vida de milhões de pessoas. Além disso, a epidemia de informação - ou "infodemia" - tornou a crise de saúde pública mais difícil de controlar e conter. O que quero dizer exatamente com "infodêmica"? Alguns fatos, misturados com medo, especulação e rumores, amplificados e retransmitidos rapidamente no mundo inteiro pelas modernas tecnologias da informação, afetaram as economias nacionais e internacionais, a política e até mesmo a segurança de formas totalmente desproporcionais às realidades básicas. É um fenômeno que temos visto com maior frequência nos últimos anos - não apenas em nossa reação à SARS, por exemplo, mas também em nossa resposta ao terrorismo... seus efeitos futuros podem ser ainda maiores. Sem controle, poderia iniciar um período de novas formas de ineficiência econômica profundas, oportunidades para os irresponsáveis e para os demagogos de praticar novas formas de perturbação ou manipulação social, e um conjunto de novos problemas sérios para os formuladores de políticas que lidam com desafios desde a saúde pública até assuntos internacionais. (Rothkopf, 2003).
\end{abstract}

Essa primeira definição é mais interessante porque inclui a dimensão do afeto. A infodemia não deveria ser tratada apenas semanticamente. As informações não representam apenas estados de coisa, vontades ou conceitos, mas expressam a tonalidade afetiva do acontecimento e do território onde o enunciado emerge e circula. No entanto, nos documentos da EPI-WIN e da OMS temos uma caracterização que se preocupa menos com a semiose afetiva dos enunciados do que com a semântica. Guardemos a definição talhada nos documentos da EPI-WIN, visto que é a determinante dos procedimentos da gestão infodêmica.

Sendo assim, acho relevante apontar a existência de uma ambiguidade nessa descrição. Dominantemente, a infodemia é concebida como a circulação excessiva de informações de todo tipo, informações confiáveis, comprovadas cientificamente, verificadas e baseadas em evidências e informações falsas, imprecisas, incorretas, 
desinformação, teorias da conspiração e/ou negacionismo. Minoritariamente, porém, lança-se mão do conceito de desinfodêmia. Em Desinfodemia, Julie Posetti e Kalina Bontcheva (2020, p.3) escrevem:

\begin{abstract}
Para entender a desinfodêmia, considera-se o seu oposto - a informação como a base para o conhecimento. É o Acessado em à informação, não à desinformação, que torna o direito à liberdade de expressão relevante e útil às sociedades. Informações comprováveis, confiáveis, como as que são produzidas pela ciência e pelo jornalismo profissional, são essenciais na construção do que a UNESCO descreve como "sociedades do conhecimento". A desinfodêmia se opõe a isso.
\end{abstract}

Não é um mero detalhe essa diferenciação, haja vista que a opção de enfatizar a desinformação eleva o nível de responsabilidade das empresas de mídia, como Facebook, Twitter e Youtube, sobretudo. A plataformização da internet é um fator sem o qual qualquer medida de enfrentamento à infodemia torna-se insuficiente, como procuro demonstrar nas seções a seguir. Porém, nesse documento, as próprias autoras chamam atenção para este fato como se vê nesta passagem: "as instituições que possibilitam essa transmissão (empresas de internet e os meios de comunicação, por exemplo) não são simples operadoras nem plataformas, mas possuem seus próprios interesses específicos em controlar e moldar o fluxo de conteúdo" (Posetti; Bontcheva, 2020, p. 3). Talvez esse seja o ponto crucial: o modelo de negócio e os algoritmos das empresas de tecnologia. Contudo, inexplicavelmente, essa questão não é explorada pelo discurso infodemiológico, como demonstro abaixo. Há ainda um outro elemento. Desinformação traduz disinformation, mas não há uma palavra precisa para traduzir misinformation, que poderia ser informação incorreta ou imprecisa. ${ }^{6} \mathrm{O}$ que marca a diferença entre os termos é a intencionalidade. Enquanto a disinformation é produzida deliberadamente para extrair efeitos políticos, a misinformation, não (Posetti; Bontcheva, 2020, p. 3).7 Constata-se, portanto, essas oscilações inerentes ao conceito de infodemia. É, afinal, um problema de dupla face. Existe uma sobreposição de infodemias. "A explosão de informações e esforços no setor humanitário se somou a um já enorme tsunami de informações, e enquanto novos esforços para combater a situação são louváveis, eles se multiplicam rapidamente, tornando a duplicação e o ruído severamente problemáticos" (WHO, 2020, p. 17). A infodemiologia nasce para lidar com esse problema.

Na seção seguinte, procuro examinar as fontes primárias do pensamento infodemiológico para tentar encontrar as tendências ali contidas, e cotejá-las, em seguida, com o trabalho da EPI-WIN.

\footnotetext{
${ }^{6}$ Em geral, nos documentos em português, informação incorreta traduz misinformation.

7 Seria preciso refletir se a intencionalidade seria o melhor critério para avaliar a desinformação. Num certo sentido, as mensagens confiáveis também têm um objetivo deliberado de influenciar e mudar comportamentos. Fico em dúvida se a intencionalidade é uma boa maneira de marcar essa diferença. De todo modo, parece ser esse o consenso. Lemos e Oliveira, por exemplo, pensando especificamente sobre a questão das fake news, a define fake news nesses termos: "Pode-se definir fake news como mensagens falsas que, embora pareçam verdadeiras, são intencionalmente produzidas com a intenção de influenciar pessoas ou grupos em prol de interesses específicos, majoritariamente políticos" (Lemos, Oliveira, 2020, p. 194).
} 


\title{
O NASCIMENTO DA INFODEMIOLOGIA
}

A infodemiologia emerge no virar do século XX para o XXI. A literatura de referência aponta para o texto inaugural de Eysenbach, Infodemiology: The Epidemiology of (Mis)information (2002). ${ }^{8}$ Neste artigo, o primeiro a conceber o termo infodemiologia, Eysenbach apresenta um ensaio do que viria a constituir a nova ciência. Faço então uma breve genealogia desse discurso, analisando como o fio do saber infodemiológico foi sendo tecido ao longo das últimas décadas até o empreendimento da EPI-WIN em formalizá-lo como a ciência de gestão infodêmica. ${ }^{9}$ Concentro a análise nos principais textos de Eysenbach por ter sido não só o proponente deste discurso, mas também um dos pioneiros a tentar implementar, ainda no início dos anos 2000, a infodemiologia junto às instituições de governança global. Depois comento os desdobramentos teórico-práticos dessa proposta em outros autores.

Eysenbach (2002, p. 1) concebe a infodemiologia assim:

\begin{abstract}
"uma nova disciplina de pesquisa e uma nova metodologia emergiu - o estudo dos determinantes e da distribuição de informação sobre saúde e de informação imprecisa - que pode ser útil para orientar profissionais da saúde e pacientes a chegar até informações sobre saúde de qualidade na internet".
\end{abstract}

A nova disciplina está vocacionada a "identificar áreas com uma lacuna de tradução de conhecimento entre as melhores evidências (o que os especialistas sabem) e a prática (o que as pessoas fazem ou acreditam), bem como de marcadores para informação de "alta-qualidade"”' (Eysenbach, 2002, p. 1). Sua proposta procurava estabelecer quais seriam os critérios para identificar e marcar os sites sobre saúde com informações confiáveis e os que contém informações sem comprovação científica.

Em 2003, Eysenbach propôs uma segunda hipótese: o uso de dados relacionados à pesquisa de palavras-chave no Google, Yahoo e Ask poderia ser testado como um instrumento de monitoramento ou vigilância de dois tipos de acontecimento: 1) a circulação de vírus ou doença; 2) ataques de bioterrorismo. As então chamadas novas tecnologias de informação e comunicação, "em contextos de um surto viral ou de um ataque de bioterrorismo, (...) podem ajudar a combinar inteligências e a detectar doenças antecipadamente" (Eysenbach, 2003, p. 3). ${ }^{10}$

Esses dois textos contêm as principais tendências da infodemiologia. Eysenbach chega a diferenciá-las dizendo que a primeira está do lado da oferta, identificando a qualidade de informação produzida, e a segunda está do lado da demanda, monitorando o comportamento do público na internet. Contudo, o discurso infodemiológico nascente acabou por privilegiar a segunda tendência. Isso ocorreu por pressão dos surtos de

\footnotetext{
8 Quatro trabalhos de revisão bibliográfica ratificam essa afirmação: Mavragani (2020), Barros et. al. (2020), Zeraatkar e Ahmadi (2018) e Bernard TM et. al (2010).

9 Vale notar que o termo infodemia não era mobilizado na primeira fase da infodemiologia e que o conceito de fake news ou correlatos e questões referente à desinformação, negacionismo e teorias conspiratórias também não apareciam. Penso ser importante ressaltar essa diferença pois ela indica a mudança de contexto político em que a discussão se coloca.

${ }^{10}$ No entanto, a alusão feita aos ataques bioterroristas desaparece dos textos posteriores de Eysenbach.
} 
influenza durante o inverno do Norte Global. Por exemplo, em 2003, a OMS convocou onze laboratórios e nove países para se juntar ao projeto de um multicentro de pesquisa para o diagnóstico da SARS. Esse trabalho foi conduzido pela Global Public Health Intelligence Network, que faz parte da Global Outbreak Alert and Reponse Network da OMS, desenvolvida e operada pela Health's Canada Centre for Emergency Preparedness and Response, que tinha como um dos responsáveis Eysenbach. ${ }^{11} \mathrm{~A}$ rede é em essência: "um rastreador especializado em detectar, na internet, artigos de notícias indicando acontecimentos inusuais relevantes à saúde pública" (Eysenbach, 2003, p. 2). O autor fala de uma tecnologia para saúde das populações:

\footnotetext{
“A tecnologia para saúde das populações é um termo guarda-chuva recente que subsumi aplicações de tecnologias tais como a Internet, aparelhos sem fio, telefonia móvel, equipamentos e casas (domotics) inteligentes que tem o foco nas populações e o potencial de aprimorar a saúde pública. A princípio, todos os aparelhos de monitoramento doméstico, desde os termômetros digitais até os aparelhos de monitoramento de asma, podem ser modificados para funcionar como um sistema de previsão, isto é, transmitir dados - via wireless ou internet - para unidades centrais de mineração de dados os quais são capazes de detectar padrões emergentes indicando surtos de doenças" (Eysenbach, 2003, p. 2).
}

Porém, como reconhece Eysenbach (2003), o uso desse procedimento precisava ser mais bem examinado, o resultado não tinha sido significante até aquele momento.

Em 2006, Eysenbach lança um paper com a hipótese que correlaciona o aumento de buscas sobre a gripe na internet, em particular nos motores do Google, e a temporada de gripe no Canadá. Demonstra que as buscas por palavras-chave no Google têm a capacidade de monitorar a circulação do vírus aproximadamente duas semanas antes dos métodos epidemiológicos tradicionais. Chama atenção que Eysenbach (2006) foi obrigada a driblar o algoritmo da plataforma, via Google Adsense, visto que a empresa não disponibilizava os dados de pesquisa nem permitia o acesso aos seus bancos de dados. Essa foi a primeira demonstração de que a infodemiologia poderia servir aos agentes de saúde pública como uma metodologia sólida para o enfrentamento de surtos patogênicos. A segunda tendência torna-se, assim, objeto de investimento da infodemiologia, sobrepondo-se à marcação da qualidade dos sites de informação sobre saúde. Por outro lado, esse procedimento carecia de uma discussão entre pares tendo em vista recortar o campo da infodemiologia e edificar um consenso em torno da sua metodologia e da sua linguagem. ${ }^{12}$

Essa discussão ganha consistência a partir de 2008. Times de pesquisadore/as de diferentes universidades se juntaram às empresas de tecnologia para experimentar

\footnotetext{
${ }^{11}$ Sempre foi um projeto de Eysenbach, mesmo antes de cunhar sua metodologia, fornecer subsídios práticos e conceituais à OMS e aos governos: "apresento um modelo concreto sobre como essa colaboração poderia ser e forneço algumas recomendações sobre qual poderia ser o papel da OMS e dos legisladores nesse quadro" (Eysenbach, 2001).

12 Note-se que isso ocorre concomitantemente à consolidação da economia digital e do capitalismo de plataforma (o Facebook é criado em 2004, o Twitter, em 2006). Economia digital e capitalismo de plataforma são termos extraídos da caracterização de Nick Snierck, em Platform capitalism (2019), em especial o segundo capítulo.
} 
metodologias semelhantes às propostas por Eysenbach. Esse debate é disparado pela publicação de dois textos de cientistas vinculados ao Yahoo e ao Google. Em 2008, pesquisadores da Universidade de lowa, Harvard e do Yahoo publicaram Using internet searches for influenza surveillance (Polgreen et al, 2008), confirmando a hipótese de que as buscas na internet, no caso feitas no Yahoo, antecipavam de uma a duas semanas a detecção da circulação do vírus H1N1. Em 2009, uma equipe do Google e do Center for Disease Control and Prevention dos E.U.A publicou Detecting influenza epidemics using search engine query data (Ginsberg et al, 2009). Nesse texto, os autores compilaram a metodologia utilizada e os resultados obtidos que permitiram a criação do Google Flu Trends. O Google Flu Trends foi uma novidade importante, pois aplica os algoritmos e a base de dados da empresa para o monitoramento do espalhamento da doença nos EUA e apresenta um dashboard para acompanhamento dos casos. ${ }^{13}$ Esses estudos são os primeiros a consolidarem a infodemiologia como uma metodologia científica comprovada e que deveria ser incluída à gestão da saúde pública. ${ }^{14}$

As pesquisas realizadas nesta década de nascimento da infodemiologia investigavam os meios adequados e a metodologia comprovadamente eficiente para municiar os governos com uma análise comportamental das populações e dos indivíduos fundamentada em uma ciência de dados ainda incipiente. Os artigos do Google e do Yahoo inauguram um debate cujo objetivo é viabilizar a implementação da infodemiologia. Por exemplo, Eysenbach é rápido em polemizar com os pesquisadores do Google, reivindicando para si a invenção da metodologia que após o estudo da Big Tech colheu notoriedade. No artigo Infodemiology and Infoveillance: framework for an emerging set of public health informatics methods to analyse search, communication and publication behavior on the internet (2009), Eysenbach repropõe uma estrutura de trabalho específica que deve se ocupar com:

\begin{abstract}
a análise de pesquisas feitas nos motores de busca para prever surtos de doença (p. ex. influenza); o monitoramento da atualização do status das pessoas em microblogs como o Twitter para a monitorar sintomas; detectar e quantificar disparidades das informações sobre saúde disponíveis; identificar e monitorar as publicações relevantes de saúde pública na internet (p. ex. sites antivacinação, mas também novos artigos e boletins organizados por especialistas); ferramentas automatizadas para medir a difusão de informação e de tradução de conhecimento, e rastrear a efetividade das campanhas de marketing de saúde. Mais ainda, a análise de como as pessoas pesquisam e navegam na internet a procura de informações relacionadas à saúde e de como elas se comunicam e compartilham informações oferecem insights valiosos sobre o
\end{abstract}

\footnotetext{
13 Sobre o conceito de dashboard, ver o excelente livro de Bratton The stack: On software and sovereignty (2015).

14 Essa passagem do texto é taxativa: "Porque as buscas podem ser processadas com rapidez, o resultado das estimativas de possíveis casos de influenza antecipavam consistentemente de uma a duas semanas os boletins de monitoramento pelo Center for Disease Control and Prevention. A detecção antecipada por essa abordagem pode tornar-se uma importante linha de defesa contra futuras epidemias de influenza nos Estados Unidos, e, talvez, eventualmente, em cenários internacionais. Estimativas atualizadas de influenza podem viabilizar que oficiais da saúde pública e profissionais da saúde a responder melhor às epidemias sazonais" (Ginsberg et al, 2009, p. 4).
} 
comportamento das populações em relação à saúde (Eysenbach, 2009, p. 2).

Dois anos depois, Eysenbach reafirma que o objetivo subjacente:

\begin{abstract}
a esse campo emergente é mensurar o pulso da opinião pública, da atenção, do comportamento, do conhecimento e atitudes por meio do rastreamento do que as pessoas fazem e escrevem na internet. O termo análise de previsão tecno-social (TPA, techno-social prediction analysis) também foi proposto, embora previsão não capte inteiramente todo o feixe de aplicações possíveis da infodemiologia (Eysenbach, 2011, p. 2).
\end{abstract}

TPA é uma metodologia elaborada por Boulos (2010). ${ }^{15} \mathrm{~A}$ crítica de Eysenbach se deve ao fato de que, em sua visão, a TPA está limitada apenas à probabilística de acontecimentos futuros (forecasting) sem entender que o que as plataformas viabilizam é uma probabilística do presente (nowcasting). Para pontuar sua diferença em relação à TPA, sugere a adição de um procedimento infodemiológico chamado de infovigilância ou infomonitoramento (infoveillance), que nada mais seria que a mineração e a análise de dados do comportamento do público nas plataformas digitais em tempo real, tomando pulso do conteúdo do que as pessoas estão dizendo, do que elas estão lendo, do que e como se informam, e qual informação compartilham em suas redes, além de analisar também quais suas opiniões e o que elas estão sentindo, sendo possível detectar qualquer mudança súbita de comportamento e de estado afetivo presentemente. Em um texto coautorado com Cyntia Chew, Eysenbach afirma:

Novos métodos de infovigilância como mineração, agregação e análise de dados textuais on-line em tempo real estão se tornando disponíveis. O Twitter é potencialmente adequado para mineração e análise longitudinal de textos. Os breves 140 caracteres de atualizações do status do texto que os usuários compartilham com "seguidores" (por exemplo, pensamentos, sentimentos, atividades, opiniões) contêm uma riqueza de dados. A mineração destes dados fornece um retrato instantâneo das opiniões e respostas comportamentais do público. O rastreamento longitudinal permite a identificação de mudanças nas opiniões ou respostas. Além da análise quantitativa, o método também permite a exploração qualitativa das razões prováveis pelas quais ocorreram mudanças repentinas (por exemplo, um relatório de notícias amplamente lido) e pode indicar o que está prendendo a atenção do público (Chew; Eysenbach, 2010, p. 2).

Começa a ficar mais evidente a confluência entre saber médico, comunicacional, informacional, e ciência comportamental e de dados. Essa confluência aparece nítida na pesquisa de Damon Centola. Centola (2013; 2018) investiga os desenvolvimentos que a análise de dados traz ao que denomina como a ciência do comportamento em saúde. A contribuição de Centola para esse debate decorre do fato de que ele

\footnotetext{
15 O objetivo do texto de Boulos era "revisar um número de tecnologias e instrumentos emergentes ... que podem ser usados para explorar as características inerentes à web social em tempo-real ou próximo a isso e lapidá-las para propósitos de vigilância em saúde pública, meio ambiente e segurança nacional” (Boulos et al, 2010, p. 17), dedicando, inclusive, uma seção para discussão dos textos de Eysenbach e do Google Flu Trends. A TPA oferece "uma racionalidade antecipatória por meio de uma abordagem multidisciplinar para uma análise e uma resposta estratégica que permite um esforço concertado para a tomada de decisão por parte de políticos e analistas" (Boulos et al, p. 19).
} 
realmente procura descrever o mecanismo que faz com que uma epidemia informacional ocorra. Para ele, isso tem que ver com a infraestrutura das redes sociotécnicas que determinam os modos pelo qual as informações proliferam em encontros casuais dos agentes dessa rede. Estudos empíricos do comportamento em saúde pré-internet enfrentaram desafios metodológicos significativos, pois são incapazes de identificar as causas dessas mudanças súbitas. Esses dados não fornecem, ou não podem fornecer, uma avaliação empírica do grau de influência direta dos vínculos da rede social. Com a plataformização das redes sociais, passa a ser possível estender esse rastreio aos vínculos fracos de uma cadeia de relações. Para Centola, a difusão de informação é mais intensa em redes sociais com vínculos fracos onde coalizões fortuitas e com uma cauda longa se formam com mais facilidade e baixo custo, em contraste com redes onde imperam vínculos fortes na qual a informação fica restrita a um circuito menos poroso. ${ }^{16}$ As plataformas digitais permitem explorar a causalidade da formação dessas coalizões por meio da extração e da análise de dados.

Haveria muito o que explorar na obra de Centola. Sua tese de um design social para intervir na dinâmica de mudança de comportamento em saúde (mas não só) é profunda e requereria mais minúcia. O fundamental, porém, para os propósitos da nossa argumentação, segue sendo o fato de que a mineração e a análise de dados determinam como e onde intervir para modular comportamentos. Esse é um tema central e que desliza pela superfície desses enunciados. Esse é o substrato que dá consistência ao discurso infodemiológico, expressando o envelopamento recíproco de saber e poder.

\section{INFODEMIOLOGIA: A CIÊNCIA DA GESTÃO DA INFODEMIA}

Inicialmente, portanto, os dois problemas infodemiológicos estavam, ao menos em tese, separados. De um lado, a epidemia de informação imprecisa ou falsa, de outro, extração e análise de dados sobre doenças e comportamentos relativos a questões de saúde. Se isso é verdade, contudo, com a pandemia da Covid-19, essas duas tendências se agenciam em uma unidade dinâmica.

Trato então de como isso é tecido nos documentos da EPI-WIN. Dois textos sintetizam as ideias, problemas, tendências e proposições engendradas na rede: Managing the Covid 19 infodemic, de setembro de 2020, e WHO public health research agenda for managing infodemics, de fevereiro de 2021. Esse material é a síntese de quatro atividades organizadas pela EPI-WIN. A consulta pública realizada nos dias 7 e 8 de abril de 2020, audiência que bateu recorde de engajamento na história da OMS. A segunda é a Primeira Conferência de Infodemiologia, que ocorreu de 30 de junho a 16 de julho

\footnotetext{
${ }^{16}$ Coiera (2013) corrobora essa hipótese: “estruturas sociais e reticulares dessas comunidades online são tão diversas quanto as estruturas sociais humanas e podem ser qualquer coisa desde fracas, abertas e oportunistas até fechadas, rígidas e secretas. O que chama atenção é a capacidade de criar coalisões fracas entre indivíduos que partilham um propósito comum de curto prazo" (Coiera, 2013, p. 1). O exemplo utilizado é digno de nota: "O papel das mídias sociais no movimento Occupy, de onde se espalharam protestos contra a desigualdade pelo mundo, e os distúrbios civis da Primavera Árabe são dois exemplos recentes" (Coiera, 2013, p. 1).
} 
daquele mesmo ano. A Conferência é um divisor, pois estrutura uma agenda comum e coordenada de pesquisa e de mobilização internacional e busca implementar um programa e uma linguagem padronizada para o novo campo. A terceira remete ao treinamento oferecido pela rede em outubro para a formação da primeira turma de gestão de infodemias da OMS. A quarta é a Terceira Conferência Virtual Global de Gestão da Infodemia, que aconteceu nos meses de outubro a dezembro de 2020, e resultou na elaboração de um chamado global para o enfrentamento da desordem informacional e de uma declaração redigida por várias instituições da sociedade civil organizada.

Nesse material, a infodemiologia coloca três problemas, contém cinco dimensões e consequentemente determina cinco áreas de atuação. Os três problemas são: 1) quais são as formas de classificar a informação imprecisa (misinformation) relacionada à saúde no que concerne o seu potencial de dano (à saúde das pessoas, comportamentos insalubres, coesão social, desconfiança na prestação de serviços de saúde, desconfiança no governo, desconfiança nas comunidades, desconfiança na mídia etc.)? 2) Como os diferentes tipos de informação imprecisa sobre saúde afetam o comportamento online e offline e quais são algumas das medidas que podem ajudar a prever o impacto dos tipos informação imprecisa sobre o comportamento? 3) Quais seriam os critérios de avaliação das respostas à infodemia caso sejam necessárias novas intervenções decorrente da COVID-19?

Dessas perguntas são desdobradas cinco dimensões da infodemiologia.

\begin{abstract}
1) A dimensão computacional será fundamental. A capacidade de aproveitar enormes quantidades e fluxos de dados terá uma diferença crítica, permitindo reações em tempo real e fechando loops de feedback; mas isso implicará em algum viés, e isso terá que ser combatido. 2) A dimensão comportamental da infodemiologia, um espaço excitante e uma fronteira atual para a concepção de políticas em geral, é central para este novo campo. 3) As dimensões cognitiva e emocional da infodemiologia interagem com as preocupações comportamentais, e os pontos de vista da neurociência cognitiva e social podem ser especialmente importantes. 4) A dimensão sociocultural da infodemiologia é complicada e as conclusões sólidas são difíceis de encontrar, mas ferramentas como análises de redes sociais e estudos antropológicos são claramente úteis. A dimensão da comunicação é crucial. Embora tenhamos uma certa compreensão do que é necessário, é preciso reconhecer que ainda não estabelecemos os conhecimentos necessários e as evidências de impacto, e novas abordagens são necessárias (WHO, 2021, p. 14).
\end{abstract}

Pode-se notar que essas cinco dimensões abordam a tecnologia, a ciência, o comportamento ou a política, a cultura, a comunicação. Dessas cinco dimensões decorrem cinco correntes de intervenção. 1) Medir e monitorar o impacto de infodemias durante emergências de saúde por meio de métricas e ferramentas padronizadas para acompanhar a evolução da infodemia no ambiente físico-digital da informação e entre indivíduos, comunidades, sociedade e o sistema de saúde, utilizando abordagens multidisciplinares, incluindo métodos e abordagens de inteligência artificial, processamento de linguagem natural, e utilizando dados 
estruturados e não estruturados (grandes dados, dados etnográficos e similares); 2) Detectar e entender a propagação e o impacto de infodemias com uma abordagem comum necessária para entender a disseminação superabundante de informações e desinformação e como isso afeta o comportamento online e offline em diferentes populações; 3) Responder e implantar intervenções que protejam contra a infodemia e atenuem seus efeitos nocivos; 4) Avaliar intervenções infodêmicas e fortalecer a resiliência de indivíduos e comunidades a infodemias e para identificar intervenções que sejam eficazes em diferentes contextos, populações, culturas e para diferentes tipos de eventos agudos de saúde; 5) Promover o desenvolvimento, adaptação e aplicação de ferramentas para a gestão de infodemias visto que existe o desejo de melhorar a transferência das lições aprendidas e intervenções baseadas em evidências entre contextos, países e infodemias.

É como se as duas tendências, a infodemiologia e a infovigilância, passassem a operar em uma lógica de inclusão mútua. Uma é condição da outra e requalifica as capacidades da outra. Nesse contato, o que caracteriza cada uma delas muda de qualidade. De um lado, a infovigilância se complexificou. A quantidade de informação e de dados é praticamente inesgotável. Segundo porque, em muito pouco tempo, as práticas disponíveis para extrair e analisar dados foram de uma gambiarra para burlar o Google Adsense ao uso de inteligência artificial e aprendizado de máquina. Ademais, acessar os dados das empresas de tecnologia é tema de conflito até então insolúvel. O modelo de negócios dessas empresas está montado para extrair lucro do agenciamento desses dados. Acessá-los é tocar nessa caixa preta. Há também o fato de que o algoritmo e o design das plataformas digitais são minuciosamente pensados para colonizar nossa atenção e nosso tempo, influenciar nosso comportamento na plataforma, tudo isso para gerar um volume cada vez maior de informação que serve para pouca coisa além de mapear nosso comportamento e nossos estados afetivos tendo em vista monetizar nossas relações e vender anúncios personalizados (Bruno, Bentes, Faltay, 2019). É verdade que as Big Techs fizeram um esforço, foram forçadas pela conjuntura a fazer um esforço, de seguir as orientações dos agentes de saúde e fornecer dados gratuitamente à OMS e a ensaiar uma agência interna, caso do Facebook, de moderação de conteúdo. Além disso, enfrentaram também pressões para suspender, deletar e mesmo impedir a publicação de conteúdo sem base em evidência, o que esbarra numa discussão abstrata, mas em todo caso importante sobre liberdade de expressão nas plataformas.

A infodemiologia também se complexificou. A checagem de fatos tornou-se a principal ferramenta de classificação das informações, algo sequer cogitado anteriormente. Claro, por conta da proliferação das notícias falsas se consolidou uma metodologia de checagem de fatos que fez com que esse procedimento fosse rapidamente adotado. A primeira iniciativa da OMS e da ONU para lidar com a infodemia foi o lançamento da Verificado. Outro procedimento é a desmitificação (myth debunking) que procura identificar o conteúdo das principais informações imprecisas e desinformações circulando nas redes e produzir um material imediatamente traduzível para várias línguas e territórios que desmascare tais mensagens. Uma terceira é a geração de textos, cartilhas, vídeos contendo evidências científicas que sejam facilmente 
traduzíveis para qualquer pessoa e que tenha a eficácia de fazê-la mudar de comportamento. Há ainda uma quarta questão, que engloba as demais, que é uma literacia digital, ou seja, estruturar uma pedagogia que consiga educar o público de como lidar com o consumo de informação nas plataformas digitais.

Está muito evidente que a infodemiologia é engendrada para oferecer uma técnica de intervenção social baseada em certos princípios e saberes tecnopolíticos (análise de dados, psicologia comportamental, cibernética). Em sua gênese, como o trabalho de Eysenbach confirma, a infodemiologia emergiu como uma metodologia voltada para identificar a oferta informacional da área da saúde na internet e para o monitoramento e a vigilância dos comportamentos de populações e indivíduos nas plataformas digitais com o objetivo de prever mudanças de hábitos e surtos epidêmicos. Atualmente, dada a nossa situação pandêmica e infodêmica, essas duas tendências convergem para dar início ao que a OMS chama de a ciência de gestão da infodemia. A infodemiologia ganha um estatuto epistemológico antes impensável, compondo o núcleo dos saberes convocados para a administração de uma crise humanitária de escala poucas vezes vistas na história do capitalismo. Tornou-se necessário articular um discurso que tivesse capacidade de entregar procedimentos comprovadamente eficientes para enfrentar uma conjuntura extremamente complexa de crises sobrepostas. A infodemia é um acontecimento que requer a instauração de um campo específico de pesquisa, com um programa de enfrentamento globalmente coordenado. Isso porque há um reconhecimento mais ou menos explicitado nas publicações e encontros da EPIWIN que muito provavelmente essa condição pandêmica veio para ficar, sendo a primeira de muitas, dado o estado de emergência climática, política e econômica atual.

O discurso infodemiológico trata muito pouco sobre o arranjo tecnopolítico das plataformas e da economia digital. É verdade que a infodemiologia se preocupa com o papel das plataformas na infodemia e demanda das empresas um compromisso para o enfrentamento do surto informacional. É verdade também que a infodemiologia reivindica $\mathrm{o}$ uso do que há de mais sofisticado no universo das máquinas computacionais. No entanto, na prática, a EPI-WIN direciona suas energias e seus recursos para gerir uma intervenção preocupada em moderação de conteúdo, literacia e, sobretudo, em distinguir o discurso verdadeiro do falso, com base em evidências científicas. É como se o que faltasse fosse uma explicação bem explicada, numa confiança quase inocente de que a razão e o bom senso das populações e indivíduos e políticos e empresas e de todas as partes interessadas nos guiarão à iluminação. Esse tipo de abordagem, além de desconsiderar toda a crítica à razão feita pelo pensamento do pós-guerra, desconsidera o modo de produção e de reprodução social que nos governa e que condiciona o surgimento da pandemia e da infodemia. Desconsidera, por fim, inexplicavelmente, o modelo de negócios das plataformas e a economia digital que a sustenta.

Como manter esse tipo de postura depois da Cambridge Analytica (CA), por exemplo? ${ }^{17}$ Insisto em dizer que os materiais da EPI-WIN não refletem criticamente sobre a lógica

17 Se paramos para ler a proposta de um dos intelectuais da CA, empresa que geriu a campanha de Trump, do plebiscito do Brexit, e, suspeita-se, de Bolsonaro, Michal Kosinski, torna-se difícil sustentar 
que fundamenta a economia digital e as plataformas de mídia. O design, o algoritmo, o modelo de negócios correspondem a uma ordem político-econômica que, como a caracteriza Zuboff (2021, p. 13), "reivindica a experiência humana como matéria-prima gratuita para práticas comerciais dissimuladas de extração, previsão e vendas". Não é possível não levar em consideração algo que está diretamente conectado com o modo como a infodemia ocorre. $O$ modo de funcionamento que é a condição de possibilidade operativa das plataformas digitais é em grande parte responsáveis pelo advento de algo como a infodemia. Além disso, nenhum desses textos medita sobre os riscos de municiar os governos com instrumentos de intervenção social com esse grau de sofisticação e capilaridade, sequer aventam a hipótese de estabelecer um critério de fiscalização institucional para essa intervenção. Tampouco refletem sobre as consequências de conservar esses dados como propriedade de empresas monopolistas. É preciso reconhecer que, como o faz Morley et. al (2020, p. 6-7), "esta falta de atenção à infosfera é incompreensível”, aceitável apenas num mundo préinternet,

quando as mídias sociais e aplicativos móveis não existiam, e quando a retórica dominante era a de que o espaço online não tinha nenhuma influência sobre as realidades físicas. No entanto, hoje em dia, as fronteiras entre online e offline são consideravelmente menos distintas e as pessoas que vivem na era da informação vivem na vida-em-linha (onlife). Parece ser hora de os órgãos de saúde pública aceitarem que a infosfera (abrangendo todas as fontes de informação on-line) tem uma influência determinante na saúde física do público.

Portanto, é preciso encarar a tecnopolítica da infosfera para compreender os comportamentos das populações, em especial, no caso, comportamentos e condutas em saúde pública. Sem observar isso, será necessário reconhecer que a ciência de gestão da infodemia comporá a malha de técnicas de intervenção social do Estado e do Mercado. Para criar os meios de refrear a proliferação da infodemia, a meu ver, é

acriticamente a postura da EPI-WIN. Kosinski propõe um psicological targeting para uma efetiva persuasão digital em massa. Diz Kosinski (2017): “A comunicação de massa persuasiva tem como objetivo encorajar grandes grupos de pessoas a acreditar e agir do ponto de vista do comunicador (...) As pesquisas sugerem que a comunicação persuasiva é particularmente eficaz quando adaptada às características e motivações psicológicas únicas das pessoas, uma abordagem que chamamos de persuasão psicológica. A proposta desta pesquisa é simples, mas poderosa (...) Pesquisas recentes no campo das ciências sociais computacionais (...) sugerem que os perfis psicológicos das pessoas podem ser previstos com precisão a partir das pegadas digitais que elas deixam com cada passo que dão on-line (...) Esta forma de avaliação psicológica a partir de pegadas digitais faz com que seja primordial estabelecer até que ponto os comportamentos de grandes grupos de pessoas podem ser influenciados através da aplicação da persuasão de massa psicológica - tanto para o seu próprio interesse (por exemplo, persuadindo-as a se alimentarem de maneira saudável) como contra os seus melhores interesses (por exemplo, persuadindoas a apostar)". Um dos textos que investigam as possiblidades da persuasão digital em massa é escrito por cientistas do Facebook. Kramer, Guilloroy e Hancock (2014) fizeram um experimento que procurou demonstrar a transferência de estados emocionais via contágio na plataforma. O artigo detalha a pesquisa realizada com 700 mil usuários divididos em dois grupos afetados por conteúdos emocionais diferentes, um era exposto a uma amostra de conteúdos positivos e o outro a conteúdos negativos. Para eles, os estados emocionais podem ser transferidos via contágio emocional, levando as pessoas a experimentar as mesmas emoções sem sua consciência. Os resultados "indicam que as emoções expressas por outros no Facebook influenciam nossas próprias emoções, constituindo uma evidência experimental de contágio em grande escala via redes sociais" (Kramer, Guilroy, Hancock, 2014). O estudo foi feito, frisa-se, sem anuência dos usuários. 
necessário tomar a infosfera pelo que ela é: um dispositivo tecnopolítico, um laboratório psicopolítico de produção de subjetividade. Não é possível negligenciar esse arranjo, por melhores que sejam as intenções (afinal, tudo é feito em nome da preservação da vida). A infodemiologia tem força para, de fato, estruturar uma comunidade de pesquisa e prática consistente para o enfrentamento da infodemia. É preciso, entretanto, considerar a rede de causalidades em sua processualidade política e histórica.

Nesse sentido, a infodemiologia conforma a trama dos enunciados de um processo que pode vir a recompor a lógica algorítmica e o modelo de negócios das plataformas digitais vigente desde o início dos anos 2000. São muitos os sinais que indicam essa reestruturação do modelo do capitalismo de plataforma. Por exemplo, em 2021, a Austrália implementou uma lei que taxa as plataformas digitais tendo em vista arrecadar recursos para o financiamento da Imprensa no país. O Facebook respondeu com um algoritmo que impede a publicação e o compartilhamento de notícias na plataforma pelos australianos. Por outro lado, o Google acatou a decisão e a Microsoft não só a apoia como faz lobby para que seja replicado em outros lugares. Outro exemplo recente é o documento publicado pela União Europeia 2030 Digital Compass (2021), no qual se explicita os fundamentos tecnopolíticos para o avanço da digitalização da sociedade europeia, conservando seus valores e sua soberania. A UE quer desenvolver uma infraestrutura continental autônoma, plataformas próprias e implementar uma economia digital soberana em relação aos EUA e à China. Dentre as principais formas de alocação de recursos para o financiamento do projeto está a taxação da economia digital. ${ }^{18}$ Por outro lado, os Estados Unidos apresentaram um projeto de lei intitulado Journalism Competition and Preservation Act of 2021 no qual tentam equiparar as condições de negociação entre a Imprensa tradicional, produtores de conteúdo, e as plataformas. ${ }^{19}$ Experimentamos uma situação de disputa aberta entre vários grupos de interesse de monta da máquina capitalista. É possível que o futuro próximo das comunicações e da economia digital estejam sendo decididos nesse emaranhado de práticas e enunciados variados, e que a infodemiologia tem força suficiente, devido sua relação com esferas não-científicas, de determinar os desdobramentos desse processo.

\section{CONCLUSÃO}

Para concluir, quero retomar a citação inicial de Machado (1978). O autor situa a relação de imanência entre saber e poder, no sentido de que o discurso da ciência, o saber científico, não deve ser objeto apenas de uma história das ideias sem passar por um exame das contaminações e transversalidades que co-emergem por uma inevitável tração genealógica com esferas não científicas, como a política, a economia, as instituições vigentes e o contexto sócio-histórico e cultural de momento. É esse

18 Acessado em: 15 mar. 2021. Disponível em: https://ec.europa.eu/info/strategy/priorities-20192024/europe-fit-digital-age/europes-digital-decade-digital-targets-2030_en

19 Acessado em: 15 mar: 2021. Disponível em: https://www.congress.gov/bill/117th-congress/senatebill/673/text 
princípio de confluência entre esferas científicas e não-científicas que tomei como critério para analisar genealogicamente o processo de nascimento do saber infodemiológico enquanto a ciência eleita para tratar um dos maiores desafios da atualidade que é a infodemia. O discurso infodemiológico, enquanto ciência, entra, assim, em uma unidade dinâmica com esferas não-científicas. Isso me parece irrefutável de constatar quando acompanhamos o desenvolvimento da disciplina, como penso ter demonstrado no estudo dos textos e documentos mobilizados nos capítulos acima. Caso me detivesse apenas no conteúdo explícito dos discursos, no aspecto apenas semântico, e na linearidade histórica dos problemas e dos conceitos infodemiológicos, perderia de vista o substrato das forças políticas moventes e interessadas a determinar e a intervir no agenciamento populacional de onde o discurso infodemiológico emerge.

Nesse sentido, procurei rastrear os principais autores e as principais tendências internas do saber infodemiológico. No entanto, não os analisei tendo como parâmetro metodológico percorrer ou traçar uma coerência interna dos discursos. Busquei, antes, estabelecer uma conexão genealógica implícita entre o nascimento da infodemiologia e os acontecimentos políticos que co-ocorriam e pressionavam em maior ou menor medida o modo de pensar infodemiológico em sua própria emergência, como, por exemplo, a primeira pandemia de influenza deste século e os eventos relacionados ao 11 de setembro. Sendo assim, destaca-se, em especial, o trabalho de Eysenbach como um dos primeiros pesquisadores a estruturar a infodemiologia enquanto uma ciência voltada a municiar as instituições de governo vigentes com as ferramentas, as práticas e os conceitos mais adequados para, por um lado, enfrentar a circulação de informação falsa e inadequada sobre saúde e, por outro, monitorar e extrair dados dos usuários para melhor compreender quais mensagens são mais convenientes para abordar determinados assuntos, e isso em um período em que a Internet não era monopolizada pelas plataformas digitais, Facebook, Twitter e Youtube sequer existiam.

Eysenbach sugere, então, os termos infodemiologia e infovigilância ou infomonitoramento para nomear a metodologia de trabalho do novo campo do saber circunscrito inicialmente a um ramo da epidemiologia, mas que com o passar dos anos viria conquistar autonomia própria. Como vimos, com o avanço da plataformização da Internet, empresas como Google e Yahoo começaram a produzir estudos direcionados à compreensão dos mesmos problemas suscitados por Eysenbach. Por estarem em posição privilegiada, ou seja, por serem proprietárias de uma enormidade de dados que circulam pela internet, essas empresas conseguiram comprovar que a infodemiologia poderia ser útil para a previsão de epidemias e como ferramenta de intervenção populacional em tempo-real para controlar o espalhamento de doenças infecciosas. Esses estudos consolidaram a metodologia e abriram um novo momento para o discurso infodemiológico, sobretudo nos países do norte mais afetados por gripes sazonais de influenza. Esse é um momento de afirmação e de consolidação da infodemiologia enquanto uma ciência comprovadamente eficiente em monitorar os comportamentos dos indivíduos e das populações nas plataformas digitais tendo em vista intervir na sociedade para extrair efeitos políticos específicos relativos ao controle do espalhamento de doenças infecciosas e de informações inadequadas. 
No entanto, tentei pontuar que a consolidação da infodemiologia ocorre entrelaçadamente com a plataformização do capitalismo e da sociedade que passa a ocorrer em meados dos anos 2000, conforme Srnicek (2019). As consequências desse processo são múltiplas, mas um efeito crucial é, justamente, a proliferação e o uso de desinformação e informações falsas para fins políticos e econômicos específicos, em uma escala planetária e intricada ao cotidiano da vida social de maneira inédita. Isso demanda um investimento coletivo, social, político, econômico e institucional para a investigação do problema, particularmente no que tange à área da saúde, tema esse caro a infodemiologia desde sua gênese. A infodemiologia volta sua lente analítica mais uma vez para os efeitos (e, curiosamente, não para as causas) da proliferação de notícias sobre a saúde nas plataformas digitais. Já se temia, ou já se intuía o dano que poderia ser causado caso uma pandemia virótica ocorresse concomitamente a uma pandemia informacional, algo explorado na literatura infodemiológica inicial sem o aprofundamento ou o investimento necessário. A pandemia do novo coronavírus é o acontecimento que força o saber infodemiológico a se debruçar inteiramente sobre o problema da infodemia.

Dado a catástrofe das milhares de mortes e da capacidade do excesso informacional de impedir que as medidas necessárias para mitigar o espalhamento do vírus e para reduzir os óbitos fossem implementadas, a ONU e a OMS declararam que paralelamente à pandemia vivíamos uma infodemia e que ela era tanto ou mais fatal. Para entender e criar os meios de lidar com a infodemia ONU e OMS criaram algumas iniciativas como a agência de checagem de fatos Verificado e, a mais importante delas, a EPI-WIN. A EPI-WIN tornou-se, dentro da OMS, o departamento responsável para estruturar e estabelecer as bases da infodemiologia. É o primeiro esforço global no sentido de estruturar e estabelecer uma comunidade de prática e de pesquisa cujo objetivo é o gerenciamento da infodemia e o objeto a infodemia. Para cumprir seu objetivo, a EPI-WIN convoca e organiza vários fóruns de debate com especialistas de várias áreas de pesquisa, dando um caráter interdisciplinar à nova disciplina. Além disso, a rede publica continuamente um rico material para alinhar uma metodologia e uma linguagem comum e replicável. A finalidade é oferecer aos Estados-membros, à sociedade e a demais agentes interessados (plataformas, mídia, mercado, comunidades religiosas, organizações não-governamentais) propostas de protocolos, medidas e políticas públicas a serem efetivadas para que seja possível uma cooperação internacional para a gestão da infodemia.

Entretanto, como tentei demonstrar ao longo do texto, está muito evidente que a infodemiologia é engendrada para oferecer uma técnica de intervenção social baseada em certos princípios e saberes tecnopolíticos (ciência de dados, psicologia comportamental, cibernética). Em sua gênese, como o trabalho de Eysenbach atesta, a infodemiologia emergiu como uma metodologia voltada para identificar a oferta informacional da área da saúde na internet e para o monitoramento e a vigilância dos comportamentos de populações e indivíduos nas plataformas digitais com o objetivo de prever mudanças de hábitos e surtos epidêmicos. Atualmente, dada a situação pandêmica e infodêmica, a infodemiologia ganha um estatuto epistemológico antes impensável, compondo o núcleo dos saberes convocados para a administração de uma 
crise humanitária de escala poucas vezes vistas na história do capitalismo. Tornou-se necessário articular um discurso que tivesse capacidade de entregar procedimentos comprovadamente eficientes para enfrentar uma conjuntura extremamente complexa de crises sobrepostas. A infodemia é um acontecimento que requer a instauração de um campo específico de pesquisa, com um programa de enfrentamento globalmente coordenado. Isso porque há um reconhecimento mais ou menos explicitado nas publicações e encontros da EPI-WIN que muito provavelmente essa condição pandêmica veio para ficar, sendo a primeira de muitas, dado o estado de emergência climática, política e econômica atual. Nesse sentido, a infodemiologia conforma a trama dos enunciados de um processo que pode vir a recompor a lógica algorítmica e o modelo de negócios das plataformas digitais que imperam desde o início dos anos 2000. Tudo isso, porém, está em aberto e vai depender das pressões e das disputas entre os distintos agentes e forças políticas interessadas social e economicamente na realização desse processo. Devemos, portanto, estudar e intervir criticamente com a finalidade de tornar esse desenvolvimento o mais democrático e aberto possível, tendo a preservação da vida como critério inegociável.

\section{REFERÊNCIAS}

BARROS et. al. 2020. The Application of Internet-Based Sources for Public HealthSurveillance (Infoveillance): Systematic Review. J Med Internet Res. Acessado em: 15 mar. 2021. Disponível em: DOI: 10.2196/13680

BERNARDO, Theresa Marie, et al. 2013. Scoping Review on Search Queries and Social Media for Disease Surveillance: A Chronology of Innovation. Journal of Medical Internet Research 15 (7): e2740. Acessado em: 10 mar. 2021. https://doi.org/10.2196/jmir.2740.

BOULOS, Maged N. Kamel, et al. 2010. Social Web mining and exploitation for serious applications: Technosocial Predictive Analytics and related technologies for public health, environmental and national security surveillance, Computer Methods and Programs in Biomedicine, Volume 100, Issue 1. Acessado em: 10 mar. 2021. Disponível: https://doi.org/10.1016/j.cmpb.2010.02.007.

BRATTON, Benjamin. 2015. The stack: On software and sovereignty. Cambridge, Massachusetts: MIT Press.

BRUNO, Fernanda; BENTES, Anna; FALTAY, Paulo. 2019. Economia psíquica dos algoritmos e laboratório de plataforma: mercado, ciência e modulação do comportamento. Revista Famecos, Porto Alegre, v. 26, $\mathrm{n}^{\circ}$ 3, set-dez. Acessado em: 12 mar. 2021. Disponível em: <http.//revistaseletronicas.pucrs.br/ojs/index.php/revistafamecos/article/view/33095>

CENTOLA, Damon. 2010. An Experimental Study of Homophilyin: the Adoption of Health Behavior. Vol. 334, Issue 6060, pp. 1269-1272. Acessado em: 10 mar. 2021. Disponível: DOI: 10.1126/science.1207055

CENTOLA, Damon, 2013. Social media and the science of health behavior. Acessado em: 10 mar. 2021. Disponível: doi.org/10.1161/CIRCULATIONAHA.112.101816.

CENTOLA, Damon, 2018. How Behavior Spreads: The Science of Complex Contagion. Princeton University Press. 
CHALHOUB, Sidney. Cidade febril: cortiços e epidemias na Corte imperial. 1996. São Paulo: Companhia da Letras.

COLETIVO CHUANG. 2020. Contágio social. São Paulo: Baderna.

European Commission. 2021. 2030 Digital Compass: the European way for the Digital Decade. Acessado em: 10 mar. 2021. Disponível em: https://ec.europa.eu/info/sites/info/files/communication-digital-compass-2030_en.pdf

EYSENBACH, Gunther. CHEW, Cynthia. 2010. Pandemics in the Age of Twitter: Content Analysis of Tweets during the 2009 H1N1 Outbreak. PLOS ONE. 29 novembro 2010. vol. 5, no. 11, p. e14118. DOI 10.1371/journal.pone.0014118.

EYSENBACH, Gunther. DIEPGEN, TL, 1998. Towards quality management of medical information on the internet: evaluation, labelling, and filtering of information. BMJ. 28 novembro 1998. vol. 317, no.

EYSENBACH, Gunther. 2002. Infodemiology: the epidemiology of (mis)information. The American Journal of Medicine. dezembro. vol. 113, no. 9, p. 763-765. DOI 10.1016/S0002-9343(02)01473-0.

EYSENBACH, Gunther. 2003. SARS and Population Health Technology. Journal of Medical Internet Research. 30 junho 2003. vol. 5, no. 2, p. e14. DOI 10.2196/jmir.5.2.e14.

EYSENBACH, Gunther. 2006. Infodemiology: Tracking Flu-Related Searches on the Web for Syndromic Surveillance. AMIA Annu Symp Pro: 244-8. Acessado em: 10 mar. 2021.Disponível em: PMID: 17238340; PMCID: PMC1839505.

EYSENBACH, Gunther. 2009. Infodemiology and Infoveillance: Framework for an Emerging Set of Public Health Informatics Methods to Analyze Search, Communication and Publication Behavior on the Internet. J Med Internet Res 2009;11(1):e11. Acessado em: 10 mar. 2021. Doi: 10.2196/jmir.1157.

EYSENBACH, Gunther. 2011. Infodemiology and infoveillance tracking online health information and cyberbehavior for public health. Am J Prev Med. Acessado em: 10 mar. 2021. doi: 10.1016/j.amepre.2011.02.006.

FOUCAULT, Michel. 1999. Vigiar e Punir. Tradução: Raquel Ramalhete. Petrópolis: Vozes.

FOUCAULT, Michel. 1973. O nascimento da clínica. Tradução: Roberto Machado. Rio de Janeiro: Forense Universitária.

FOUCAULT, Michel. 2006. O poder psiquiátrico. Curso do Collège de France (19731974). Tradução de Eduardo Brandão. São Paulo: Marins Fontes.

GINSBERG, J. et al. 2009. Detecting influenza epidemics using search engine query data. Natur, 1012-1014. Acessado em: 10 mar. 2021. Disponível em: https://doi.org/10.1038/nature07634.

KOSINSK. Michal, et al. 2017. Psychological targeting in digital mass persuasion. Proceedings of the National Academy of Sciences. Acessado em: 10 mar. 2021. 
Disponível em: DOI: 10.1073/pnas.1710966114

KRAMER, A. D. I., J. E. GUILLORY, and J. T. HANCOCK. 2014. "Experimental Evidence of Massive-Scale Emotional Contagion through Social Networks." Proceedings of the National Academy of Sciences 111 (24): 8788-90. https://doi.org/10.1073/pnas.1320040111.

LEMOS, André. OLIVEIRA, Frederico. 2020. Fake news no Whatsapp. um estudo da percepção dos efeitos em terceiros. C\&S - São Bernardo do Campo, v. 42, n. 1, p. 193227, jan.-abr. Acessado em em: 15 mar. 2021. Disponível em: https://doi.org/10.15603/2175-7755/cs.v42n1p193-227.

MACHADO, Roberto. 1978. Danação da norma: medicina social e constituição da psiquiatria no Brasil. Rio de Janeiro: Graal.

MAVRAGANI, A. 2021. Infodemiology and Infoveillance: Scoping Review. J Med Internet Res. Acessado em: 10 mar. 2021. DOI: 10.2196/16206.

MORLEY, Jessica, et al. 2020. "Public Health in the Information Age: Recognizing the Infosphere as a Social Determinant of Health." Journal of Medical Internet Research. Acessado em: 10 mar. 2021. https://doi.org/10.2196/19311.

Organização Pan-Americana da Saúde. 2020. Entenda a infodemiae a desinformaçãona luta contra a COVID-19. Acessado em: 10 mar. 2021. Disponível em: https://iris.paho.org/bitstream/handle/10665.2/52054/Factsheet-

Infodemic_por.pdf?sequence=14

POLGREEN, Philip M., et al. 2008. Using Internet Searches for Influenza Surveillance. Clinical Infectious Diseases, vol. 47, no. 11, p. 1443-1448. Acessado em: 15 mar. 2021. Disponível: www.jstor.org/stable/40308150.

POSETTI, Julie, BONTCHEVA, Kalina. 2020. Desinfodemia: Decifrar a desinformação sobre a COVID-19. International Center for Journalists (ICFJ). Genebra: UNESCO. Acessado em 20 mar. 2021. Disponível em: https://en.unesco.org/covid19/disinfodemic.

ROTHKOFP, David. 2002. When the buzz bites back. Acessado em: 20 mar. 2021. Disponível em: http.//www1.udel.edu/globalagenda/2004/student/readings/infodemic.html

SRNICEK, Nick. 2017. Platform capitalism. Polity Press.

Staff, Reuters. 2021. Factbox: How U.S. Bills Would Help News Media Negotiate with Facebook, Google. Acessado em mar. 10, 2021. Disponível em: https://www.reuters.com/article/us-tech-antitrust-media-factbox-idUSKBN2B22NC.

TANGCHAROENSATHIEN, Viroj. et al. 2020. Framework for Managing the COVID-19 Infodemic: Methods and Results of an Online, Crowdsourced WHO Technical Consultation". Journal of Medical Internet Research 22 (6): e19659. Acessado: 10 mar. Disponível em: https://doi.org/10.2196/19659.

ZERAATKLAR, Kimia; AHMADI, Maryam. 2018. Trends of infodemiology studies: a scoping review. Health Information \& Libraries Journal, (), -. doi:10.1111/hir.12216 . 
ZUBOFF, Shoshana. 2021. A era do capitalismo de vigilância. Rio de Janeiro: Intrínseca.

World Health Organization. 2020. An ad hoc WHO technical consultation managing the COVID-19 infodemic: call for action, 7-8 April 2020. Geneva: World Health Organization; 2020.

World Health Organization. 2021. WHO public health research agenda for managing infodemics. Geneva: World Health Organization. 